\title{
Fabrication of Dense Shoulder Components through Traveling Zone Sintering Assisted by a Multi-Way Loading System*
}

\author{
Shuji Tada, Zheng Ming Sun, Hitoshi Hashimoto and Toshihiko Abe \\ Institute for Structural and Engineering Materials, National Institute of Advanced Industrial Science and Technology, \\ Sendai 983-8551, Japan
}

Dense forming of shoulder components through the pulse discharge sintering process was examined. A new technique of multi-way loading system is proposed. This loading system is able to control the pressure in a powder compact body equally even if it has an irregular sectional profile. The traveling zone heating method combined with this loading system will allow the successful sintering of shoulder components through the PDS process, since no sinking due to pressure difference occurs in a powder compact. Based on this idea, the conventional sintering procedure with a perforated spacer was applied to production of a shoulder component. The spacer dictated the loading stroke on the thin side. The result was that an aluminum shoulder component with diameters of $\phi 15 \mathrm{~mm}$ at the thin side and $\phi 25 \mathrm{~mm}$ at the thick side was satisfactorily produced. The relative density of the component exceeded $99 \%$. Some problems remain to be solved, but this procedure has potential for achieving high reproducibility by developing a precise load control system and stable contact between the electrode and the cylinder.

(Received October 16, 2003; Accepted December 22, 2003)

Keywords: sintering, traveling zone heating, shoulder component, load control, aluminum powder, density

\section{Introduction}

Pressurized pulse discharge sintering, PDS, (sometimes called spark plasma sintering or SPS) is a very useful process for forming components from refractory materials, since it requires less manufacturing energy than either casting processes or conventional sintering methods such as hot pressing or hot isostatic pressing. ${ }^{1)}$ Many studies have been reported on this sintering process for the fabrication of machine components, ${ }^{2,3)}$ especially engine parts that require high heat resistance. ${ }^{4)}$

In the PDS process, however, there are several difficulties with precise temperature control, since the process heats the whole material compact body at once. The variation in temperature distribution within the powder compact aggravates as it becomes longer, especially if it has irregular sectional profiles. This may result in uneven and unsatisfactory quality of sintered components.

In order to improve the quality of products sintered using the PDS process, the authors proposed a traveling zone sintering method, ${ }^{5)}$ in which electric power was supplied to a limited zone perpendicular to the loading axis through a slidable electrode. The electrode was able to travel along the sidewall of the cylinder in the direction of the loading axis while remaining in continuous contact with it. This method could fine-tune the sintering temperature at each position, making it possible to produce long rods of high quality. At the same time, the loading problem for components with irregular sectional profiles has not been solved. The pressure applied to the material compact is inversely proportional to its sectional area. Hence, it was very difficult to maintain the desired form, since the thin side of the compact sinks into the thick side very easily during sintering due to the pressure difference between them.

In the present investigation, a new loading system for the

*This Paper was Presented at the Autumn Meeting of the Japan Institute of Metals, held in Sapporo, on October 13, 2003
PDS process was examined with the aim of preventing materials from deforming during sintering. Based on the traveling zone heating method, dense sintering procedure of shoulder components was studied via the approach of pressure control within the powder compact.

\section{The Concept of Multi-Way Loading Sintering}

Figure 1 is a schematic illustration of the load control procedure for a shoulder sample in the PDS process. The material powder compact placed in a mold is usually pressurized from the top end by a single loading ram, Ram A. There is in fact some friction between the powder compact and the inner wall of the mold, but it is ignored here. Under these conditions, the pressure in the compact is inversely proportional to the sectional area, with the result that the pressure at the thick side, $\boldsymbol{P} \boldsymbol{b}$, falls from that at the thin side $\boldsymbol{P a}$ in proportion to the enlargement of its sectional area. Consequently, it is very difficult, in the conventional PDS

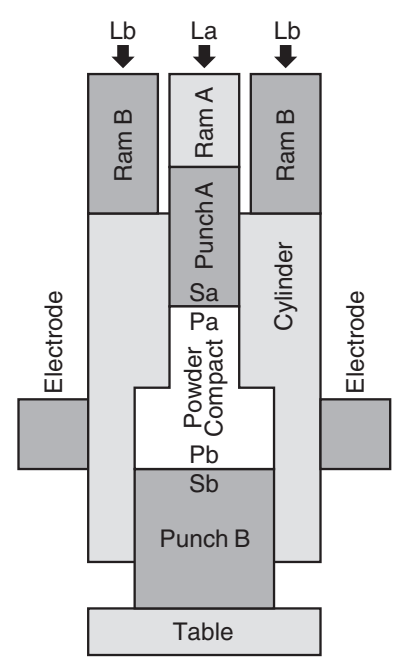

Fig. 1 A schematic illustration of the two-way loading system. 
process, to maintain the desired shoulder form, since the thin side easily sinks into the thick side during sintering.

Conversely, the two-way loading system, in which two individual loading rams are applied, is able to match these different pressures. In this loading system, Ram A pressurizes the powder compact through Punch A while Ram B presses the mold directly, leading to skip loading to the thick side. This means that the pressure on the thin side $\boldsymbol{P a}$ is determined by Ram A only, while that at the thick side, $\boldsymbol{P} \boldsymbol{b}$, comprises a combination of Ram A and Ram B. The pressures $\boldsymbol{P a}$ and $\boldsymbol{P} \boldsymbol{b}$ in the powder compact are thus given by

$$
\begin{aligned}
P a & =L a / S a \\
P b & =(L a+L b) / S b
\end{aligned}
$$

where $\boldsymbol{S a}$ and $\boldsymbol{S} \boldsymbol{b}$ are, respectively, the sectional areas at the thin side and the thick side, and $\boldsymbol{L} \boldsymbol{a}$ and $\boldsymbol{L} \boldsymbol{b}$ are loads applied by Ram A and Ram B. From eqs. (1) and (2), it is obvious that $\boldsymbol{P} \boldsymbol{b}$ can be made to match $\boldsymbol{P} \boldsymbol{a}$ by adjusting $\boldsymbol{L} \boldsymbol{b}$ under the following conditions,

$$
L b=L a(S b-S a) / S a
$$

This loading system allows the successful sintering of shoulder components through the PDS process, since no sinking due to pressure difference occurs in the powder compact. This loading system may also be suitable for multistepped components by increasing the number of loading rams in accordance with the steps in the product to be sintered.

\section{Experimental Procedure}

The material used was $99.9 \%$ pure atomized aluminum powder with an average diameter of $20 \mu \mathrm{m}$. A cylindrical mold was employed in this study to produce a shoulder component. The bore of the cylinder was $\phi 15 \mathrm{~mm}$ on the thin side and $\phi 25 \mathrm{~mm}$ on the thick side. The width of the electrode which could delineate the heating zone was $15 \mathrm{~mm}$. Both the cylinder and the punches were made from ISO-68 grade graphite distributed by Toyo Tanso Co., Ltd.

$4.77 \mathrm{~g}$ or $9.54 \mathrm{~g}$ of aluminum powder was placed on the thin side of a cylinder to give target lengths of $10 \mathrm{~mm}$ and $20 \mathrm{~mm}$, respectively while the thick side was filled with $13.25 \mathrm{~g}$ of aluminum powder for the densified length of $10 \mathrm{~mm}$.

Sintering was started from the thick side. The sintering machine used was, unfortunately, equipped with only a single loading ram. We then prepared a perforated spacer through which the punch passed so as to release the pressure in the thin side. The spacer was able to set the loading stroke on the thin side. Figure 2 illustrates this loading system. In the primary stage of sintering, the load was transmitted to the powder compact through Punch A. Electric power was supplied to the thick side but shrinkage was mainly caused in the thin side due to the pressure difference, so sinking was more noticeable in Punch A rather than in Punch B. Where the loading ram touched the spacer, the load was released from Punch A but passed to the cylinder. This loading system enabled pressurized sintering on the thick side. It is important that the thickness of the spacer be slightly thicker than the

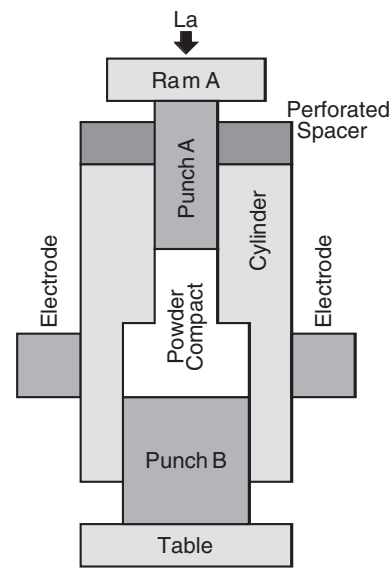

Fig. 2 Load-relaxing sintering method for a shoulder component assisted by a perforated spacer.

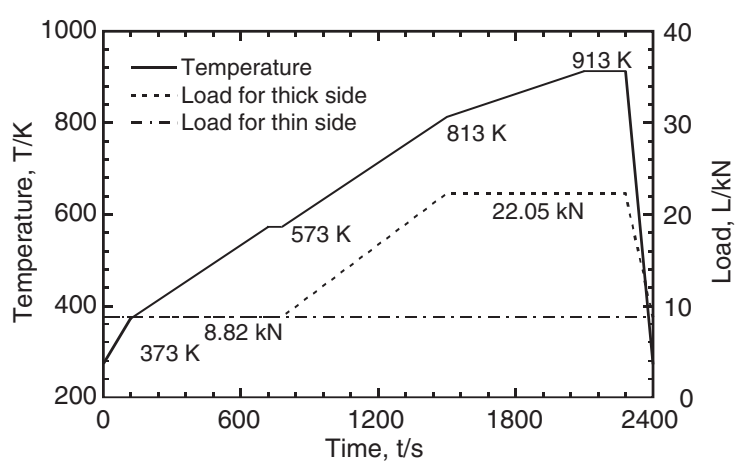

Fig. 3 Loading and heating diagram for sintering of shoulder component.

expected shrinkage on the thin side. Too thick a spacer will cause reverse sinking to the powder compact. The next sintering stage was prepared by moving the electrode to the thin side and removing the spacer. Sintering of the shoulder component was completed by electric power supplied to the thin side.

Figure 3 shows the heating and loading diagrams. Sintering pressure was conditioned to $45 \mathrm{MPa}$ for the thick side and $50 \mathrm{MPa}$ for the thin side. Temperature was monitored by a thermocouple placed just beside the powder compact. The sintering temperature was set at $913 \mathrm{~K}$.

All experiments were carried out in an inert gas atmosphere under nitrogen gas flow. The properties of the sintered samples were evaluated by examining their density using Archimedes' principle.

\section{Results and Discussion}

Figure 4 indicates the shrinking behavior of the powder compact under zone heating to the thick side. The volume of filled powder on the thin side was $4.77 \mathrm{~g}$. It is seen that the shrinkage grew as the temperature increased but it slowed down briefly at around $580 \mathrm{~K}$. This retardation is probably attributable to the loading ram flange coming into contact with the spacer. No more load was taken by the upper punch, so the shrinking was limited to only the thick side. The shrinking reaccelerated with the elevation of both temperature and the applied load. Through the above procedure, a 


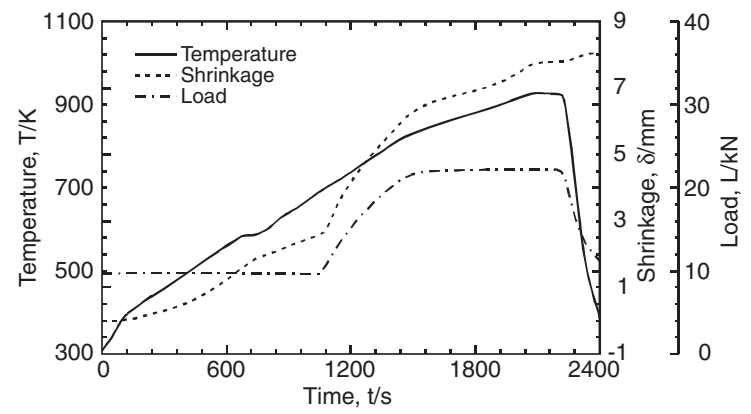

Fig. 4 Shrinking behavior in shoulder compact during heating of the thick side.

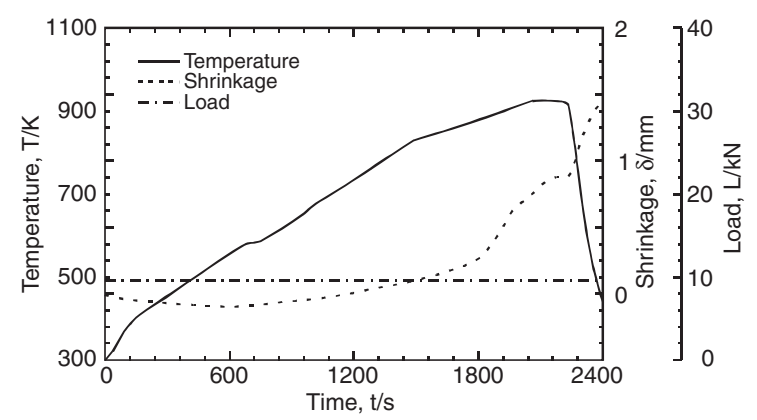

Fig. 5 Shrinking behavior in shoulder compact during heating of the thin side.

provisional shoulder component with long dimensions of $12 \mathrm{~mm}$ and $10 \mathrm{~mm}$ as the thin and the thick side, respectively, was obtained. The contraction to $10 \mathrm{~mm}$ long on the thick side means that this part was successfully densified by this method and that the sinking of the thin side was successfully restrained.

The shrinking behavior in the secondary zone heating to the thin side is shown in Fig. 5. During initial heating, some expansion was observed. This phenomenon was due to the thermal expansion of the punches more than counteracting the shrinkage of the powder compact. Shrinking returned at $560 \mathrm{~K}$ and accelerated from $880 \mathrm{~K}$.

Figure 6 displays an aluminum shoulder sample sintered using the above procedure. The length of the thin side has shrunk to $10 \mathrm{~mm}$. The relative density of this sample was $99.6 \%$, guaranteeing that the sintered body was successfully densified. Another sample, produced without a spacer, is also displayed in the same figure. It is obvious that the thin side sank into the thick side and failed to show the desired form. The above results show that the new sintering technique proposed in this study is very useful in producing densified

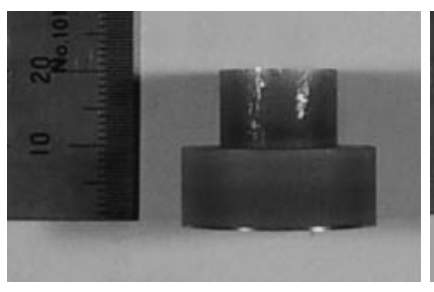

a) with spacer

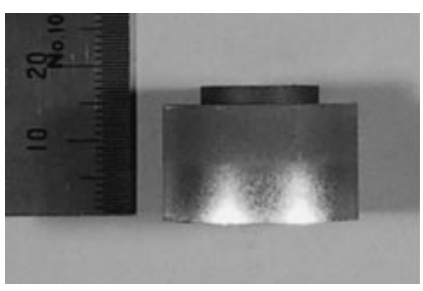

b) without spacer
Fig. 6 Aluminum shoulder components produced through the PDS process, a) with spacer, b) without spacer.

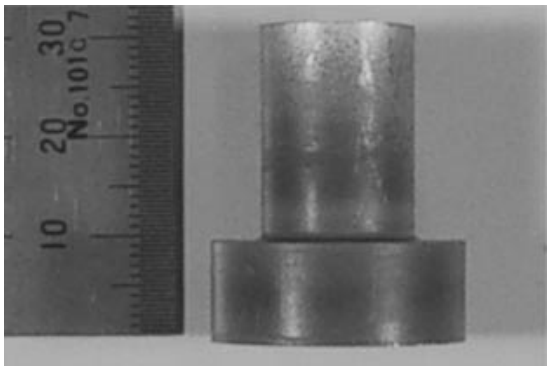

Fig. 7 Longer shoulder component produced through the PDS process when assisted by a spacer.

shoulder components through the PDS process.

A longer shoulder sample, $20 \mathrm{~mm}$ long on the thin side, was produced to examine the scale effect in this process. Electric power supplies had been planned once to the thick side and twice to the thin side for this sintering, but a cylinder cracked during the second heating. The crack originated from the insertion hole of the thermocouple. Although zone heating was continued to $913 \mathrm{~K}$ at that time, it could not proceed to the final round. In spite of this accident, the sintered sample shown in Fig. 7 reached a relative density of 99.2\%. This result can be explained as follows; the thin side was almost completely covered by the heating zone, since the width of the electrode was $15 \mathrm{~mm}$ compared to the length of the thin side of $20 \mathrm{~mm}$. Failure in the mold can be avoided by employing a suitable design for the cylinder.

In the present study, a convenient PDS process is proposed in order to produce dense shoulder components. In this method, an introduced spacer sets the loading stroke for the thin side. Estimation of its thickness is therefore very important, since too thick a spacer may cause deformation due to reverse sinking from the thick side to the thin side. Also, the conditions of contact between the electrode and the cylinder deteriorated with heating due to burnout of the cylinder surface. Moreover, it is better to place the thermocouple just on the cylinder surface in order to preserve the sliding function of the electrode.

There are still some problems to be worked out, but the traveling zone sintering method for dense shoulder components has potential to achieve high reproducibility after further development of the multi-ram operation system to give precise load control, and split electrodes which promise more stable contact between the electrode and the cylinder.

\section{Concluding Remarks}

In this study, dense sintering of the shoulder component through the pulse discharge process was examined. The obtained results are summarized as follows.

(1) Based on the traveling zone heating method, a new pressurized pulse discharge sintering technique is proposed. This load-relaxing process can produce high-density sintered machine parts with irregular sectional profiles.

(2) A spacer installed into the pulse discharge sintering system to decide the loading stroke for the thin side successfully enabled the sintering of an aluminum shoulder component with a diameter of $\phi 15 \mathrm{~mm}$ on the thin side and $\phi 25 \mathrm{~mm}$ on the thick side at a relative density exceeding $99 \%$. 
(3) The traveling zone sintering method for dense shoulder components has the potential to achieve high reproducibility after further development of the multi-ram operating system for precise load control and the split electrode which promises more stable contact between the electrodes and the cylinder.

\section{Acknowledgements}

The authors sincerely thank Ms. Harumi Ohmiya for her help in the sintering experiment and data analysis.

\section{REFERENCES}

1) M. Ohmori and T. Hirai: New Ceramics 7 (1994) 23-26 (in Japanese).

2) Z. H. Shan, Z. M. Sun, H. Hashimoto, Y. H. Park and T. Abe: Mater. Trans., JIM 40 (1999) 957-960.

3) T. Y. Um, Y. H. Park, H. Hashimoto, S. Sumi and R. Watanabe: Powder and Powder Metall. 43 (1996) 438-443 (in Japanese).

4) T. Abe, H. Hashimoto, T. Y. Um, Y. H. Park and Z. M. Sun: Proc. 1998 Powder Metallurgy World Congress, (European powder Metallurgy Association, 1998), Vol. 5, 437-442.

5) S. Tada, Z. M. Sun, H. Hashimoto and T. Abe: Mater. Trans. 44 (2003) $1667-1670$ 